\title{
Gastos públicos envolvidos no tratamento de neoplasias da coluna vertebral e medula espinhal em cidades de Minas Gerais
}

\author{
Governmental expenditure involved with the treatment of vertebral spine and spinal cord \\ neoplasms in cities of Minas Gerais
}

\author{
Enzo Pacelli Santos Fonseca ${ }^{\text {a }}$ \\ Orcid: https://orcid.org/0000-0003-2886-6933
}

João Víctor Cordeiro Rodrigues ${ }^{b}$

Orcid: https://orcid.org/0000-0002-1876-774X

\author{
Estela Silva Lopes ${ }^{c}$ \\ Orcid: https://orcid.org/0000-0003-3737-6549
}

André Samuel De Souza ${ }^{d}$

Orcid: https://orcid.org/0000-0003-2711-3702

César Felipe Gusmão Santiago ${ }^{\mathrm{e}}$

Orcid: https://orcid.org/0000-0002-0139-967X

\begin{abstract}
Resumo
Introdução: $\mathrm{O}$ diagnóstico e tratamento das neoplasias do sistema nervoso têm mostrado aumento da relevância epidemiológica nos sistemas de saúde. Apesar disso, há pouco material científico sobre os impactos financeiros dessas neoplasias. Objetivo: Analisar os gastos públicos envolvidos no tratamento de neoplasias da coluna vertebral e medula espinhal nas cidades de Belo Horizonte, Juiz de Fora, Uberlândia e Montes Claros, pertencentes a Minas Gerais, Brasil. Materiais e Métodos: Pesquisa descritiva, sistemática, transversal e quantitativa. Dados coletados referentes a 2008-2017, sobre gastos públicos com procedimentos hospitalares relacionados às neoplasias na plataforma DATASUS. Amostra composta pela população dos quatro municípios estudados acometida pelas neoplasias. Indivíduos de ambos os sexos residentes nesses locais, acometidos pela afecção, de todas as idades foram incluídos. Resultados e Conclusões: A cidade de Belo Horizonte teve gasto total de $\mathrm{R} \$ 2.069 .823,04$ com procedimentos relacionados às neoplasias da coluna vertebral e medula espinhal, enquanto Juiz de Fora, Uberlândia e Montes Claros, tiveram, respectivamente, $\mathrm{R} \$ 240.481,51, \mathrm{R} \$ 165.057,45$ e $\mathrm{R} \$ 432.930,98$. Tais gastos corresponderam a $0,05 \%$ do total de gastos hospitalares envolvidos no tratamento de todas as doenças em Belo Horizonte. A porcentagem direcionada por Juiz de Fora, Uberlândia e Montes Claros, foi, de, respectivamente: $0,028 \% ; 0,0238 \%$ e $0,0657 \%$. Portanto, o impacto econômico para o sistema público de saúde comparado ao gasto total é mínimo. Entretanto, é evidente a influência das neoplasias na taxa de mortalidade e nos anos potenciais de vida perdidos. Logo, os indicadores de saúde contribuem para que o governo crie medidas governamentais coerentes, embasadas e mais eficientes.
\end{abstract}

Palavras-chave: neoplasias; medula espinhal; coluna vertebral; gastos em saúde; hospitalização.

\footnotetext{
a Centro Universitário FIPMoc (UNIFIPMoc); Montes Claros; Minas Gerais; Brasil. E-mail: enzo.pacelli@hotmail.com

${ }^{\mathrm{b}}$ Centro Universitário FIPMoc (UNIFIPMoc); Montes Claros; Minas Gerais; Brasil. E-mail:

cordeirorodrigues.jv@gmail.com

${ }^{\mathrm{c}}$ Centro Universitário FIPMoc (UNIFIPMoc); Montes Claros; Minas Gerais; Brasil. E-mail: estelasilopes@hotmail.com

d Centro Universitário FIPMoc (UNIFIPMoc); Montes Claros; Minas Gerais; Brasil. E-mail: andresamuell1@yahoo.com

${ }^{e}$ Graduação em Medicina. Neurocirurgião e preceptor da residência de Neurocirurgia do Hospital Santa Casa da Misericórdia de Montes Claros; Montes Claros; Minas Gerais; Brasil. E-mail: cesarscbh@gmail.com
} 


\begin{abstract}
Introduction: Diagnosis and treatment of neoplasms of the nervous system has shown increased epidemiological relevance in health systems. Despite this, there is little scientific material on the financial impacts of these neoplasms. Objective: To analyze the public expenses involved in the treatment of spinal and spinal cord neoplasms in the cities of Belo Horizonte, Juiz de Fora, Uberlândia and Montes Claros, belonging to Minas Gerais, Brazil. Materials and Methods: Descriptive, systematic, cross-sectional and quantitative research. Data collected from 2008-2017 on public expenditure on hospital procedures related to neoplasms in the DATASUS platform. Sample composed by the population of the four studied municipalities affected by the neoplasms. Individuals of both sexes residing in these places, affected by the disease, of all ages were included. Results and Conclusions: The city of Belo Horizonte spent R \$2,069,823.04 on procedures related to spinal and spinal cord neoplasms, while Juiz de Fora, Uberlândia and Montes Claros, respectively, had total spent of R \$ $240,481.51, \mathrm{R} \$ 165,057.45$ and $\mathrm{R} \$ 432,930.98$. Such expenditure corresponded to $0.05 \%$ of the total hospital expenses involved in the treatment of all diseases in Belo Horizonte. The percentage directed by Juiz de Fora, Uberlândia and Montes Claros was, respectively: $0.028 \%$, $0.0238 \%$ and $0.0657 \%$. Therefore, the economic impact on the public health system is minimal. However, the influence of neoplasms on mortality rate and potential lost years of life is evident. Thus, health indicators help the government to create coherent, informed and more efficient government measures.
\end{abstract}

Keywords: neoplasms; spinal cord; vertebral spine; health expenses; hospitalization.

\section{Introdução}

As neoplasias da coluna vertebral e medula espinhal recebem classificação macroscópica de acordo com a localização em dois tipos: tumores extradurais (mais prevalentes), entre o tecido meníngeo da dura-máter e tecido ósseo da coluna vertebral e tumores intradurais extramedular e intramedular ${ }^{1}$. As metástases ósseas são as principais neoplasias que acometem a medula espinhal e coluna vertebral, acometendo majoritariamente o segmento extradural, em mais de $95 \%$ dos $\operatorname{casos}^{2,3}$.

Dentre os tumores primários, $80 \%$ deles são representados pelos gliomas, nos quais os astrocitomas são cerca de 60 a $70 \%$ e os ependimomas 30 a $40 \%{ }^{4,5}$. Além disso, os tumores não metastáticos subdurais podem ser intramedulares - astrocitomas (mais comuns), hemangioblastomas e os ependimomas (que também pode acometer o segmento extramedular) - e em tumores extramedulares ${ }^{6}$. Nesse cenário, os meningiomas, os ependimomas intradurais extramedulares e os schwanomas intradurais extramedulares constituem 0 segundo tipo de neoplasia mais frequente ${ }^{7,8}$.
Por fim, os astrocitomas e os ependimomas intradurais intramedulares configuram os tipos mais raros de neoplasias de medula espinhal ${ }^{3,6,7}$. Essas categorias neoplásicas, inclusive, acarretam elevada morbimortalidade para os pacientes, visto que causam uma rápida deterioração neurológica e decréscimo da qualidade de vida e, por serem raros, frequentemente são subdiagnosticados, retardando o tratamento e diminuindo as chances de cura ${ }^{6,9}$.

Em relação ao tratamento, o controle local e o alívio dos sintomas são princípios primordiais. Por conseguinte, a abordagem terapêutica não deve comprometer o estado de vida do paciente, visando atenuar a expansão da doença, obter o controle da dor e reestabelecer o estado neurológico, evitando ao máximo a iatrogenia ${ }^{2}$.

A abordagem dessas neoplasias deve ser em abrangência multidisciplinar, pautada por uma equipe constituída por cirurgiões de colunas, oncologistas, radioterapeutas, radiologistas, patologistas e profissionais de enfermagem ${ }^{10}$. Além disso, deve-se considerar o paciente em conjunto com seus desejos e anseios, escolhendo a melhor terapia e atendendo a individualidade de cada caso ${ }^{11}$. 
O avanço radioterápico no tratamento dessas neoplasias é indiscutível ${ }^{3}$. Todavia, a cirurgia permanece relevante no tratamento das metástases espinhais, principalmente as cirurgias minimamente invasivas que apresentam menor morbimortalidade quando comparada à cirurgia conservadora ${ }^{11}$.

Considerando as abordagens cirúrgicas existentes, elas apresentam bom prognóstico no tratamento de pacientes com metástases espinhais sintomáticas, sobretudo se realizadas com técnicas adequadas $^{2}$. Ainda, o avanço de cirurgias minimamente invasivas garante melhor integridade da coluna vertebral, fato que reduz a dor e acelera a recuperação pósoperatória $^{12}$. E o tratamento cirúrgico para pacientes com as metástases de coluna, utilizando instrumentação pedicular e descompressão, por exemplo, pode beneficiar bastante clinicamente, sobretudo melhorando os sintomas neurológicos $\mathrm{e}$ também diminuindo a dor ${ }^{13}$.

O tratamento das neoplasias do sistema nervoso, com o avanço das técnicas de diagnóstico que objetivam uma terapia mais adequada, vem ganhando relevância epidemiológica nos sistemas de saúde ${ }^{14}$. Apesar disso, há pouco material científico sobre os impactos financeiros das neoplasias, de modo que se torna indispensável um trabalho que aborde minuciosamente informações acerca dos gastos com o tratamento desses tumores. Dessa forma, este trabalho objetiva analisar os gastos públicos envolvidos no tratamento das neoplasias da medula espinhal e coluna vertebral nas cidades de Montes Claros, Juiz de Fora, Belo Horizonte e Uberlândia, pertencentes ao estado de Minas Gerais.

\section{Metodologia}

Pesquisa descritiva, sistemática, com delineamento transversal na coleta de dados e abordagem quantitativa desses, acerca dos gastos hospitalares com neoplasias da coluna vertebral e medula espinhal em Montes Claros, Juiz de Fora, Belo Horizonte e Uberlândia, sendo todos os municípios pertencentes ao estado de Minas Gerais.

A pesquisa foi iniciada com uma revisão narrativa com enfoque nos aspectos gerais referentes à epidemiologia, diagnóstico e tratamento dessas afecções. Em seguida, coletaram-se os dados referentes aos gastos hospitalares com as neoplasias de coluna vertebral e medula espinhal, na plataforma DATASUS, abarcando os anos de 2008 a 2017. O período da coleta ocorreu no mês de fevereiro de 2019.

Com relação aos dados obtidos, a plataforma DATASUS estratifica os dados referentes ao tratamento dos tumores em: microcirurgia de tumor intradural e extramedular; microcirurgia de tumor medular com técnica complementar; microcirurgia de tumor medular e ressecção de tumor raquimedular extradural. Assim, foram coletados esses dados específicos do tratamento dessas neoplasias, bem como as informações referentes a todas as despesas com procedimentos hospitalares nesses quatro municípios.

A amostra foi composta pelos indivíduos acometidos pelas enfermidades, que fizeram tratamento ou gerou algum gasto decorrente dessas doenças nos hospitais vinculados ao SUS dessas quatro cidades. Nessa amostragem, os critérios de inclusão foram todos os indivíduos do sexo feminino e masculino que geraram algum gasto hospitalar acometidos pelas neoplasias de coluna vertebral e medula espinhal, de todas as idades, residentes ou não nesses municípios, registrados na plataforma utilizada como base do trabalho. Não houve critérios de exclusão.

A aprovação do Comitê de Ética foi dispensada nessa pesquisa por se tratar de dados de característica aberta, impossibilitando a identificação dos sujeitos, conforme a Resolução 466/12 do Conselho Nacional de Saúde.

Os resultados encontrados foram registrados e analisados através de planilhas 
eletrônicas (Excel), para melhor seleção e comparação das informações coletadas, possibilitando a construção da discussão e resultados da pesquisa.

\section{Resultados}

Os procedimentos hospitalares relacionados às neoplasias da coluna vertebral e medula espinhal, como microcirurgia de tumor intradural e extramedular, microcirurgia de tumor medular com técnica complementar, microcirurgia de tumor medular e ressecção de tumor raquimedular extradural somaram, no período de 2008 a 2017, para Belo Horizonte, Juiz de Fora, Uberlândia e Montes Claros, um gasto total de, respectivamente, $\quad \mathrm{R} \$ 2.069 .823,04$; $\mathrm{R} \$ 240.481,51 ; \quad \mathrm{R} \$ 165.057,45$; $\mathrm{R} \$ 432.930,98$, conforme os dados da Tabela 01.

Tabela 01 - Gastos, em reais (R\$), com procedimentos relacionados a neoplasias da coluna vertebral e medula espinhal em Belo Horizonte- MG, Juiz de Fora - MG, Uberlândia-MG e Montes Claros-MG

\begin{tabular}{rcccc}
\hline ANO & BELO HORIZONTE & JUIZ DE FORA & UBERLÂNDIA & $\begin{array}{c}\text { MONTES } \\
\text { CLAROS }\end{array}$ \\
\hline $\mathbf{2 0 0 8}$ & $128.312,92$ & $18.512,59$ & $25.047,89$ & $19.019,58$ \\
$\mathbf{2 0 0 9}$ & $194.912,06$ & $25.402,33$ & $13.152,14$ & $53.236,08$ \\
$\mathbf{2 0 1 0}$ & $220.638,34$ & $37.384,80$ & $28.420,31$ & $32.030,68$ \\
$\mathbf{2 0 1 1}$ & $214.245,92$ & $46.634,72$ & $13.771,52$ & $56.236,15$ \\
$\mathbf{2 0 1 2}$ & $153.040,88$ & $24.252,19$ & $26.226,71$ & $51.016,64$ \\
$\mathbf{2 0 1 3}$ & $216.609,51$ & $17.963,73$ & $3.565,24$ & $57.072,28$ \\
$\mathbf{2 0 1 4}$ & $290.546,91$ & $12.444,15$ & $19.090,90$ & $51.047,57$ \\
$\mathbf{2 0 1 5}$ & $183.014,84$ & $22.934,53$ & $8.966,69$ & $67.431,29$ \\
$\mathbf{2 0 1 6}$ & $208.955,39$ & $16.893,32$ & $6.126,54$ & $39.362,42$ \\
$\mathbf{2 0 1 7}$ & $259.546,27$ & $18.059,15$ & $20.689,51$ & $6.478,29$ \\
TOTAL & $2.069 .823,04$ & $240.481,51$ & $165.057,45$ & $432.930,98$ \\
\hline
\end{tabular}

Fonte: Ministério da Saúde - Sistema de Informações Hospitalares do SUS (SIH/SUS)

Nota-se que no período analisado, a cidade de Belo Horizonte apresentou um gasto médio de R $\$ 206.982,30$. Entre os anos de 2012 e 2013, houve o maior aumento nas despesas hospitalares $(\mathrm{n}=41,5 \%)$, passando, respectivamente, de $\mathrm{R} \$ 153.040,88$ para $\mathrm{R} \$ 216.609,51$. O menor registro foi no ano de 2008 $(\mathrm{n}=\mathrm{R} \$ 128.312,92)$, ao passo que o ano de maior despesa foi 2014, apresentando um total de $\mathrm{R} \$ 290.546,91$ gastos.

A cidade de Montes Claros no período de 2008 a 2017 apresentou um gasto total de $\mathrm{R} \$ 432.930,98$, que representa um gasto médio anual de $\mathrm{R} \$ 43.293,09$. A maior variação nos gastos corresponde à transição do ano de 2008 para 2009, com porcentagem de aumento de $180 \%$, período que houve um aumento de $\mathrm{R} \$ 19.019,58$ para $\mathrm{R} \$ 53.236,08$. O ano de menor gasto para a cidade foi 2017 , com $\mathrm{R} \$ 6.478,29$ gastos, e o de maior despesa foi 2015, com um registro de $\mathrm{R} \$ 67.431,29$.

O município Juiz de Fora, no período estudado, evidenciou um gasto hospitalar médio anual de $\mathrm{R} \$ 24.048,15$. A maior variação ocorreu entre 2014 e 2015 , uma mudança de aproximadamente $84 \%$, período no qual a cidade saiu de um gasto de $\mathrm{R} \$ 12.444,15$, alcançando o valor de $\mathrm{R} \$ 22.934,53$. O ano de menor registro foi $2014 \quad(\mathrm{n}=\mathrm{R} \$ 12.444,15)$ e o mais dispendioso para a cidade foi 2011 $(\mathrm{n}=\mathrm{R} \$ 46.634,72)$.

A cidade de Uberlândia, nesse período, evidenciou um gasto médio anual de $\mathrm{R} \$ 16.505,74$, tendo em vista que apresentou $\mathrm{R} \$ 165.057,45$ totais gastos. $\mathrm{O}$ período em que se verificam os maiores aumentos de gastos com as doenças na cidade corresponde à transição de 2009 para 2010 com aumento de $\mathrm{R} \$ 13.152,14$ para 
$\mathrm{R} \$ 28.420,31$ ( $\mathrm{n}=116 \%)$. O ano de menor registro na cidade foi o de 2013 $(\mathrm{n}=\mathrm{R} \$ 3.565,24)$ e o de maior despesa foi $2010(\mathrm{n}=\mathrm{R} \$ 28.420,31)$.

A pesquisa buscou, ainda, abordar a relação de todas as despesas hospitalares no período de 2008 a 2017, conforme pode ser observado nos dados da Tabela 02, a fim de averiguar o impacto real das despesas com procedimentos envolvidos com as doenças para os cofres públicos dessas quatro cidades.

A cidade de Belo Horizonte, durante o período, apresentou uma despesa total de $\mathrm{R} \$ 4.187 .434 .313,26$. Isso configura direcionamento de $0,05 \%$ dos gastos hospitalares totais para o tratamento, já que a cidade apresentou $R \$ 2.069 .823,04$ gastos. O município de Juiz de Fora, por sua vez, evidenciou uma despesa de $\mathrm{R} \$ 861.838 .665,10$, de modo que os gastos com as neoplasias correspondem a $0,028 \%$ desse total.

Já, a cidade de Uberlândia apresentou um gasto de $\mathrm{R} \$ 692.577 .462,00$, ao passo que em Montes Claros evidenciouse um total de $\mathrm{R} \$ 658.660 .148,44$. O valor gasto com as neoplasias de coluna vertebral e medula espinhal corresponde a $0,0238 \% \mathrm{e}$ $0,0657 \%$ do gasto total nessas duas cidades, respectivamente.

\section{Discussão}

Para que o tratamento das neoplasias possa acontecer de maneira adequada, é necessária uma estratégia diagnóstica e investigativa refinada, o que demanda gastos. $\mathrm{O}$ crescimento exponencial na utilização de terapias direcionadas, a disponibilidade de exames de imagem, como a ressonância magnética e tomografia por emissão de pósitrons, propiciaram uma melhoria considerável na sobrevida de praticamente todos os pacientes portadores de tumores da coluna vertebral e medula espinhal ${ }^{12}$.

Dados do INCA demonstram que embora tenha no Brasil auxílio do Sistema Único de Saúde (SUS), pacientes portadores de neoplasias, em geral, sofrem bastante aguardando consultas, exames e, principalmente, tratamento, ao passo que pacientes com planos de saúde lidam com a demora para autorização dos convênios que, muitas vezes, bloqueiam tratamentos e medicamentos mais custosos ${ }^{12}$.

Considerando essa perspectiva de análise de custos, verificou-se que, no período de 2008 a 2017, a cidade de Belo Horizonte apresentou o maior gasto com procedimentos relacionados às neoplasias da coluna vertebral e medula espinhal, conforme mostrado na Tabela 02. Isso ocorre por ser a capital do estado e, ainda, atender uma demanda maior de pacientes, o que implica diretamente em uma elevação nos gastos. De acordo com os dados da Tabela 03, observa-se que a capital do estado apresentou o maior número de internações para a realização de procedimentos para o tratamento dessas enfermidades. A metrópole apresentou 595 internações, ao passo que as demais cidades juntas apresentaram $222(\mathrm{n}=27,1 \%$ do total no período estudado), sendo que Montes Claros apresentou $125 \quad(\mathrm{n}=15,2 \%)$, Uberlândia $44(n=5,3 \%)$ e Juiz de Fora 53 $(\mathrm{n}=6,04 \%)$.

Tabela 02 - Gastos hospitalares totais, em reais (R\$), em Belo Horizonte - MG, Juiz de Fora - MG, Uberlândia -

\begin{tabular}{ccccc}
\multicolumn{5}{c}{ MG e Montes Claros - MG } \\
\hline ANO & BELO HORIZONTE & JUIZ DE FORA & UBERLÂNDIA & MONTES CLAROS \\
\hline $\mathbf{2 0 0 8}$ & $288.813 .123,88$ & $78.668 .481,68$ & $45.478 .872,85$ & $42.428 .493,54$ \\
$\mathbf{2 0 0 9}$ & $326.549 .075,85$ & $69.935 .082,11$ & $52.194 .653,43$ & $52.732 .253,17$ \\
$\mathbf{2 0 1 0}$ & $351.255 .638,42$ & $76.409 .419,15$ & $54.089 .973,20$ & $54.287 .523,58$ \\
$\mathbf{2 0 1 1}$ & $375.136 .717,73$ & $89.520 .416,36$ & $65.396 .593,80$ & $64.168 .466,24$ \\
$\mathbf{2 0 1 2}$ & $406.762 .857,07$ & $88.880 .579,78$ & $73.256 .561,46$ & $66.999 .293,18$ \\
$\mathbf{2 0 1 3}$ & $446.688 .542,85$ & $90.751 .043,67$ & $82.502 .014,21$ & $73.547 .921,40$ \\
$\mathbf{2 0 1 4}$ & $480.444 .328,90$ & $90.780 .138,49$ & $86.912 .823,10$ & $78.485 .683,26$ \\
\hline
\end{tabular}




\begin{tabular}{ccccc}
\hline ANO & BELO HORIZONTE & JUIZ DE FORA & UBERLÂNDIA & MONTES CLAROS \\
\hline $\mathbf{2 0 1 5}$ & $513.962 .946,62$ & $90.667 .273,4^{\prime} 1$ & $77.707 .303,19$ & $75.721 .817,18$ \\
$\mathbf{2 0 1 6}$ & $508.356 .465,08$ & $94.151 .639,21$ & $72.652 .352,81$ & $75.376 .225,15$ \\
$\mathbf{2 0 1 7}$ & $489.464 .616,86$ & $95.558 .957,04$ & $82.386 .313,98$ & $72.523 .958,49$ \\
TOTAL & $4.187 .434 .313,26$ & $861.838 .665,10$ & $692.577 .462,00$ & $658.660 .148,44$ \\
\hline \multicolumn{7}{c}{ Fonte: Ministério da Saúde - Sistema de Informações Hospitalares do SUS (SIH/SUS) }
\end{tabular}

Fonte: Ministério da Saúde - Sistema de Informações Hospitalares do SUS (SIH/SUS)

Tabela 03 - Internações por ano de processamento, segundo município, para a realização de procedimentos para o tratamento de neoplasias da coluna vertebral e medula espinhal

\begin{tabular}{ccccc}
\hline \multirow{2}{*}{ ANO } & \multicolumn{4}{c}{ MUNÍCIPIO } \\
\cline { 2 - 5 } & $\begin{array}{c}\text { BELO } \\
\text { HORIZONTE }\end{array}$ & $\begin{array}{c}\text { MONTES } \\
\text { CLAROS }\end{array}$ & UBERLÂNDIA & JUIZ DE FORA \\
\hline $\mathbf{2 0 0 8}$ & 48 & 6 & 10 & 5 \\
$\mathbf{2 0 0 9}$ & 61 & 17 & 3 & 9 \\
$\mathbf{2 0 1 0}$ & 70 & 9 & 7 & 8 \\
$\mathbf{2 0 1 1}$ & 57 & 16 & 4 & 8 \\
$\mathbf{2 0 1 2}$ & 53 & 12 & 4 & 6 \\
$\mathbf{2 0 1 3}$ & 48 & 12 & 2 & 3 \\
$\mathbf{2 0 1 4}$ & 66 & 19 & 5 & 4 \\
$\mathbf{2 0 1 5}$ & 64 & 19 & 2 & 2 \\
$\mathbf{2 0 1 6}$ & 52 & 12 & 2 & 4 \\
$\mathbf{2 0 1 7}$ & 76 & 3 & 5 & 53 \\
TOTAL & 595 & 125 & 44 & 4 \\
\hline
\end{tabular}

Fonte: Ministério da Saúde - Sistema de Informações Hospitalares do SUS (SIH/SUS)

Destaca-se, ainda, que a capital mineira apresentou maior média de permanência $(n=799,6)$ nos hospitais para a realização de procedimentos quando comparada a Montes Claros $(\mathrm{n}=220,19)$, Juiz de Fora $(n=86,8)$ e Uberlândia $(n=$ 56,2) no período de 2008-2017. Esses dados contribuem para justificar o maior gasto no município belo-horizontino, tendo em vista o alto número de internações associado a uma média de permanência alta, implica maiores gastos em termos de alimentação, higienizações, procedimentos e exames ${ }^{16}$.

A análise dos gastos hospitalares, além de ratificar a justificativa acerca do maior dispêndio aos cofres públicos no município de Belo Horizonte, também permite a verificação dos períodos de maior gasto, sendo que em 2010, 2011, 2014 e 2015 houve o maior gasto com procedimentos relacionados às doenças em Uberlândia, Juiz de Fora, Belo Horizonte e Montes Claros, respectivamente. Em Juiz de Fora o gasto de $\mathrm{R} \$ 240.481,51$ correspondeu a $11,61 \%$ do valor gasto em Belo Horizonte $(\mathrm{n}=\mathrm{R} \$ 2.069 .823,04)$, Uberlândia, por sua vez, representou 7,97\% $(\mathrm{n}=\mathrm{R} \$ 165.057,45)$ e em Montes Claros 20,91\% ( $n=R \$ 432.930,98)$, sendo essa, portanto, a segunda cidade mais dispendiosa dentre as estudadas.

O entendimento desses dados evidencia a disparidade entre a capital e as demais regiões e ainda ressalta o Norte de Minas com alto gasto frente à Zona da Mata e ao Triângulo Mineiro. Tal fato se explica por fatores regionais, uma vez que Montes Claros atua como referência no Norte de Minas para a realização de procedimentos hospitalares complexos, recebendo fluxos populacionais provenientes da mesorregião Norte, do Vale do Jequitinhonha, Vale do Mucuri e também do Sul do estado da Bahia $^{17}$.

Contudo, a análise pelo viés dos gastos hospitalares totais mostra baixa variação percentual entre as cidades Juiz de Fora, Montes Claros e Uberlândia, as quais, juntas, apresentaram uma média de R\$ 737.692.091,85, sendo que Juiz de Fora obteve 0 maior gasto $(\mathrm{n}=\mathrm{R} \$ 861.838 .665,10)$, ao passo que Uberlândia e Montes Claros apresentaram $\mathrm{R} \$ 692.577 .462,00$ e $\mathrm{R} \$ 658.660 .148,44$, o que corresponde a $80,4 \%$ e $76,4 \%$ dos gastos da primeira cidade, nesta ordem.

Ao se fazer a comparação desses dados com os gastos em Belo Horizonte, a 
variação em Juiz de Fora apresenta um percentual de $20,58 \%$ em relação aos gastos da capital, Uberlândia $15,73 \%$ e Montes Claros $16,53 \%$, fato que demonstra um valor irrisório das três cidades frente ao da capital. Esse achado pode ser explicado por diversos fatores, que variam desde a realização de procedimentos de maior complexidade, como também pelo fato de o número de internações em Belo Horizonte $(\mathrm{n}=595)$, segundo os dados da Tabela 03, ter sido cerca de 10 vezes maior que em Uberlândia $(\mathrm{n}=44)$ e Juiz de Fora $(\mathrm{n}=53)$ e, aproximadamente, 4,5 vezes maior que em Montes Claros $(\mathrm{n}=125)$.

\section{Conclusão}

A análise do cenário dos procedimentos envolvidos com o tratamento dos tumores da coluna vertebral e medula espinhal em Belo Horizonte, Montes Claros, Uberlândia e Juiz de Fora demonstra que, embora se trate de procedimentos muito complexos, o impacto para o sistema público de saúde é mínimo, sobretudo ao observar o baixo número de internações nas cidades mais interioranas, já que o número expressivo se deu apenas na capital do estado.

Todavia, é evidente a influência que essas neoplasias exercem para o estado de
Minas Gerais e para o Brasil, sobretudo no que diz respeito à taxa de mortalidade e nos anos potenciais de vida perdidos de um indivíduo, principalmente quando se considera que as metástases, principal representante dessas neoplasias, têm origem a partir de um câncer prévio, podendo acometer, portanto, as mais diversas faixas etárias, levando a um comprometimento da capacidade laborativa de um indivíduo, bem como determinando a redução da expectativa de vida.

Sendo assim, os indicadores de saúde disponíveis no banco de dados do DATASUS podem contribuir para que o governo crie, a partir dessas informações, medidas governamentais coerentes, embasadas e mais eficientes, a fim de que possa executar ações que atenuem, de alguma forma, as consequências desse tipo de patologia, para os pacientes e para o sistema de saúde como um todo. Tem-se expectativa, atualmente, de que através dos investimentos em saúde, sobretudo no que se relaciona à gestão dos serviços como, por exemplo, construção da linha de cuidado do paciente oncológico, o desenvolvimento natural da patologia possa ser modificado, o que propicia uma longevidade ampliada dos pacientes diagnosticados com essas doenças ${ }^{18}$.

\section{Referências}

1. Medrano MF, Garza PA. Astrocitoma de grau III intramedular em paciente de 21 meses: descrição de um caso. Revista Pediatria Atenção Primária 2015; 17(66), e133-e136.

2. Joaquim AF, Ghizoni E, Tedeschi H, Pereira EB, Giacomini LA. Radiocirurgia estereotáxica para metástases de coluna vertebral: revisão de literatura. Einstein (São Paulo) 2013; 11(2):247-255.

3. Joaquim AF, Maturana FAP, Anderle DV, Zambelli HJL, Maldaun MVC. Metástases na coluna vertebral. Revista Neurociências 2007; 15(3):240-245.

4. Grimm S, Chamberlain M C. Adult primary spinal cord tumors. Journal Expert Review of Neurotherapeutics 2009; 9(10):1487-1495.

5. Duong L M, McCarthy B J, McLendon RE, Dolecek TA, Kruchko C, Douglas LL, Ajani Ua. Descriptive epidemiology of malignant and nonmalignant primary spinal cord, spinal meninges, and cauda equina tumors, United States, 2004-2007. Cancer 2012; 118(17):42204227.

6. Mechtler L L, Nandigam K. Spinal Cord tumors: new viewsand future directions. Neurologic Clinics 2013; 31(1):241-268. 
7. Batista M, Pina R, Fonseca I, Saldannha MH. Ependimoma intramedular: Revisão da literatura A propósito de um caso clínico. Casos Clínicos em Medicina Interna 2009, 16(3):147-151.

8. Koeller KK, Rosenblum RS, Morrison AL. Neoplasms of the Spinal Cord and Filum Terminale: Radiologic-Pathologic Correlation. AFIP ARCHIVES 2000, 20(6):1721-1749.

9. Fisher CG, Goldschlager T, Boriani S, Varga PP, Fehlings MG, Bilsky MH, Dekutoski MB, Luzzati A, Williams RP, Berven S, Chou D, Reynolds JJ, Quraishi NA, Rhines LD, Bettegowda C, Gokaslan ZL. A novel scientific model for rare and of tenn eglected neoplastic conditions. Evid Based Spine Care J 2013; 4(2):160-162.

10. Falavigna A, Rigghesso NO, Ioppi AEE, Grasselli J. Metástases do Segmento Torácico e Lombar da Coluna Vertebral. Arquivos Neuropsiquiatria 2011; 65(3B):889-895.

11. Barzilai O, Fisher, CG, Bilsky MH. State of the Art Treatment of Spinal Metastatic Disease. Neurosurgery 2018; 82(6):757-769.

12. Choi D, Bilsky MB, Fehlings M, Fisher C, Gokaslan Z. Spine Oncology - Metastatic Spine Tumors. Neurosurgery 2017; 80(3S):S131-S137.

13. Neto JAN, Fontes BPC, Macedo RD'A, Simões CE. Patients with spinal metastasis submitted to neurological decompression and stabilization. Coluna/Columna 2016; 15(3): 230-234.

14. Brasil JPN, Takayanagui OM. Tratado de Neurologia da Academia Brasileira de Neurologia. $1^{\text {a }}$ Edição. Rio de Janeiro: Elsevier; 2013. p.1239.

15. Silva DKV, Pessoa ET, Veloso HH. Dificuldades Financeiras e seus Impactos no Tratamento de Pacientes com Câncer: uma Realidade Vivenciada no Projeto Ero. Revista Saúde \& Ciência (online) 2018; 7(2):157-169.

16. Melione LPR, Mello-Jorge MHP. Gastos do Sistema Único de Saúde com internações por causas externas em São José dos Campos, São Paulo, Brasil. Cadernos de Saúde Pública 2008; 24(8):1814-1824.

17. Filho OBA, Rigotti JIR, Campos J. Os níveis hierárquicos das cidades médias de Minas Gerais. Revista Ra'e Ga 2007; 13:7-18.

18. Brasil - Ministério da Saúde. Gabinete do Ministro. Portaria no 874, de 16 de maio de 2013. Institui a Política Nacional para a Prevenção e Controle de Neoplasias na Rede de Atenção à Saúde das Pessoas com Doenças Crônicas no âmbito do Sistema Único de Saúde (SUS). Gabinete do Ministro, 2013; 16 de maio.

\section{Como citar este artigo:}

Fonseca EPS, Rodrigues JVC, Lopes ES, de Souza AS, Santiago CFG. Gastos públicos envolvidos no tratamento de neoplasias da coluna vertebral e medula espinhal em cidades de Minas Gerais. Rev. Aten. Saúde. 2020; 18(64): 52-59. 